\title{
Asymptotic inequalities for alternating harmonics
}

\author{
Vito Lampret \\ Faculty of Civil and Geodetic Engineering \\ University of Ljubljana \\ Jamova cesta 2, 1000 Ljubljana, Slovenia \\ vito.lampret@guest.arnes.si
}

Received 5 May 2017

Accepted 15 August 2017

Published 19 March 2019

Communicated by Ari Laptev

For $n \in \mathbb{N}$ the $n$th alternating harmonic number

$$
H_{n}^{*}:=\sum_{k=1}^{n}(-1)^{k-1} \frac{1}{k}
$$

is given in the form

$$
H_{n}^{*}=\ln 2+\frac{(-1)^{n+1}}{4\left\lfloor\frac{n+1}{2}\right\rfloor}+\sum_{i=1}^{q-1} \frac{\left(4^{i}-1\right) B_{2 i}}{(2 i)\left(2\left\lfloor\frac{n+1}{2}\right\rfloor\right)^{2 i}}+r_{q}(n),
$$

where $q \in \mathbb{N}$ is a parameter controlling the magnitude of the error term $r_{q}(n)$ estimated as

$$
0<(-1)^{q+1} r_{q}(n)<\frac{\left|B_{2 q}\right|}{2 q \cdot\left\lfloor\frac{n+1}{2}\right\rfloor^{2 q}}<2 \frac{\exp \left(\frac{1}{24 q}\right)}{1-2 \cdot 4^{-q}} \sqrt{\frac{\pi}{q}}\left(\frac{q}{e \pi\left\lfloor\frac{n+1}{2}\right\rfloor}\right)^{2 q} .
$$

Keywords: Approximation; double inequality; estimate; alternating harmonic number.

Mathematics Subject Classification: Primary: 41A60; Secondary: 33F05, 65B10

\section{Introduction}

Recently, the $n$th harmonic number (shortly the $n$th harmonic) ${ }^{\dagger}$

$$
H_{n}:=\sum_{k=1}^{n} \frac{1}{k} \quad(n \geq 0)
$$

${ }^{\dagger}$ By definition, we have $\sum_{i=m}^{n} x_{i}=0$ for $m>n$, consequently $H_{0}:=0$.

This is an Open Access article published by World Scientific Publishing Company. It is distributed under the terms of the Creative Commons Attribution 4.0 (CC-BY) License. Further distribution of this work is permitted, provided the original work is properly cited. 


\section{Lampret}

was accurately estimated in [2]. Twelve years ago, it was shown in [5] that for the $n$th alternating harmonic number (shortly the $n$th alternating harmonic),

$$
H_{n}^{*}:=\sum_{k=1}^{n}(-1)^{k-1} \frac{1}{k},
$$

the best constants $a$ and $b$, satisfying the double inequality

$$
\frac{1}{2 n+a} \leq\left|H_{\infty}^{*}-H_{n}^{*}\right| \leq \frac{1}{2 n+b}
$$

for every $n \in \mathbb{N}$, are $a=\frac{1}{2 n-\ln 2}$ and $b=1$. Another proof with the best constants $a$ and $b$ can be found in [3]. Recently, the inequalities

$$
\frac{1}{4 n^{2}+2}<\left|H_{n}^{*}-(-1)^{n-1} \frac{1}{2 n}-H_{\infty}^{*}\right|<\frac{1}{4 n^{2}+1.177 \ldots} \quad(n \in \mathbb{N}),
$$

were presented in 4 which enable one to estimate the alternating harmonic numbers. We shall give some further extremely accurate estimates based on results presented in [1]2].

\section{Preliminaries}

Grouping positive and negative summands, for any $p \in \mathbb{N}$, we have

$$
H_{2 p}^{*}=\sum_{j=1}^{p} \frac{1}{2 j-1}-\frac{1}{2} \sum_{j=1}^{p} \frac{1}{j}=\left(H_{2 p}-\frac{1}{2} H_{p}\right)-\frac{1}{2} H_{p}=H_{2 p}-H_{p}
$$

and consequently

$$
H_{2 p+1}^{*}=H_{2 p}^{*}+\frac{1}{2 p+1}=H_{2 p}-H_{p}+\frac{1}{2 p+1}=H_{2 p+1}-H_{p} .
$$

Thus, using the floor (integer part) function $\lfloor\cdot\rfloor$, we have

$$
H_{n}^{*}=H_{n}-H_{\lfloor n / 2\rfloor} \quad(n \geq 1) .
$$

Now, we are in a position to use the two basic results from [1, 2].

Lemma 1 ([2, Theorem 1]). For positive integers $m, n$ and $q$, where $m<n$, we have

$$
H_{n}=A_{q}(m)+B_{q}(n)+R_{q}(m, n),
$$

where $e^{\ddagger}$

$$
\begin{gathered}
A_{q}(m):=H_{m}-\ln m-\frac{1}{2 m}+\sum_{i=1}^{q-1} \frac{B_{2 i}}{(2 i) m^{2 i}} \\
B_{q}(n):=\ln n+\frac{1}{2 n}-\sum_{i=1}^{q-1} \frac{B_{2 i}}{(2 i) n^{2 i}}
\end{gathered}
$$

$\ddagger$ See footnote ${ }^{\dagger}$. 


$$
0 \leq(-1)^{q+1} R_{q}(m, n)<\frac{\left|B_{2 q}\right|}{2 q \cdot m^{2 q}}<2 \frac{\exp \left(\frac{1}{24 q}\right)}{1-2 \cdot 4^{-q}} \sqrt{\frac{\pi}{q}}\left(\frac{q}{e \pi m}\right)^{2 q} .
$$

Next, setting $a=1$ in [1, Theorem 1], we obtain for the Euler-Mascheroni constant $C:=\gamma(1)$ the expression ${ }^{\S}$

$$
C=H_{n}-\ln (1+n)+\frac{1}{2(1+n)}+\sum_{j=1}^{q-1} \frac{B_{2 j}}{2 j(1+n)^{2 j}}+R_{n}^{*}(1, q) \quad(n, q \in \mathbb{N})
$$

with the inequalities

$$
\frac{\left|B_{2 q}\right|}{2 q(2+n)^{2 q}}<(-1)^{q+1} R_{n}^{*}(1, q)<\left(1-4^{-q}\right) \frac{\left|B_{2 q}\right|}{q(1+n)^{2 q}} .
$$

Now, considering the identity $H_{n+1}=H_{n}+\frac{1}{n+1}$, replacing $1+n$ by $n$, and introducing the notation $r_{n}^{*}(q):=-R_{n}^{*}(1, q)$, we get from relations (91) and (10) the following crucial lemma.

Lemma 2 ([1, Theorem 1]). For every integer $n \geq 1$ there holds the equality

$$
H_{n}=C+\ln n+\frac{1}{2 n}-\sum_{j=1}^{q-1} \frac{B_{2 j}}{(2 j) n^{2 j}}+r_{n}^{*}(q),
$$

with the estimates

$$
-\left(1-4^{-q}\right) \frac{\left|B_{2 q}\right|}{q n^{2 q}}<(-1)^{q+1} r_{n}^{*}(q)<-\frac{\left|B_{2 q}\right|}{2 q(n+1)^{2 q}} .
$$

\section{Approximating the Alternating Harmonics}

For any integer $n \geq 2$, using (4) and setting $m=\left\lfloor\frac{n}{2}\right\rfloor$ in Lemma 1 we obtain

$$
\begin{aligned}
H_{n}^{*}= & \left(A_{q}\left(\left\lfloor\frac{n}{2}\right\rfloor\right)+B_{q}(n)+R_{q}\left(\left\lfloor\frac{n}{2}\right\rfloor, n\right)\right)-H_{\lfloor n / 2\rfloor} \\
= & \left(\underline{H}_{\lfloor n / 2\rfloor}-\ln \left\lfloor\frac{n}{2}\right\rfloor-\frac{1}{2\left\lfloor\frac{n}{2}\right\rfloor}+\sum_{i=1}^{q-1} \frac{B_{2 i}}{(2 i)\left\lfloor\frac{n}{2}\right\rfloor^{2 i}}\right. \\
& \left.+\ln n+\frac{1}{2 n}-\sum_{i=1}^{q-1} \frac{B_{2 i}}{(2 i) n^{2 i}}+R_{q}\left(\left\lfloor\frac{n}{2}\right\rfloor, n\right)\right)-\underline{H}_{\lfloor n / 2\rfloor} \\
= & \ln \frac{n}{\lfloor n / 2\rfloor}+\frac{1}{2}\left(\frac{1}{n}-\frac{1}{\lfloor n / 2\rfloor}\right)+\sum_{i=1}^{q-1} \frac{B_{2 i}}{(2 i)}\left(\frac{1}{\lfloor n / 2\rfloor^{2 i}}-\frac{1}{n^{2 i}}\right) \\
& +R_{q}\left(\left\lfloor\frac{n}{2}\right\rfloor, n\right),
\end{aligned}
$$

$\S$ Consider footnote ${ }^{\dagger}$. 


\section{Lampret}

where using (8),

$$
0 \leq(-1)^{q+1}<R_{q}\left(\left\lfloor\frac{n}{2}\right\rfloor, n\right)<\frac{\left|B_{2 q}\right|}{2 q \cdot\lfloor n / 2\rfloor^{2 q}} .
$$

Similarly, for every integer $n \geq 2$, considering (4) and Lemma 2]

$$
\begin{aligned}
H_{n}^{*}= & \left(\boldsymbol{Q}+\ln n+\frac{1}{2 n}-\sum_{j=1}^{q-1} \frac{B_{2 j}}{(2 j) n^{2 j}}+r_{n}^{*}(q)\right) \\
& -\left(\boldsymbol{Q}+\ln \lfloor n / 2\rfloor+\frac{1}{2\lfloor n / 2\rfloor}-\sum_{j=1}^{q-1} \frac{B_{2 j}}{(2 j)\lfloor n / 2\rfloor^{2 j}}+r_{\lfloor n / 2\rfloor}^{*}(q)\right) \\
= & \ln \frac{n}{\lfloor n / 2\rfloor}+\frac{1}{2}\left(\frac{1}{n}-\frac{1}{\lfloor n / 2\rfloor}\right)+\sum_{j=1}^{q-1} \frac{B_{2 j}}{2 j}\left(\frac{1}{\lfloor n / 2\rfloor^{2 j}}-\frac{1}{n^{2 j}}\right)+r_{n}^{* *}(q),
\end{aligned}
$$

with $r_{n}^{* *}(q):=r_{n}^{*}(q)-r_{\lfloor n / 2\rfloor}^{*}(q)$.

Referring to (12),

$$
-\left(1-4^{-q}\right) \frac{\left|B_{2 q}\right|}{q n^{2 q}}<(-1)^{q+1} r_{n}^{*}(q)<-\frac{\left|B_{2 q}\right|}{2 q(n+1)^{2 q}}
$$

and

$$
\frac{\left|B_{2 q}\right|}{2 q(\lfloor n / 2\rfloor+1)^{2 q}}<-(-1)^{q+1} r_{\lfloor n / 2\rfloor}^{*}(q)<\left(1-4^{-q}\right) \frac{\left|B_{2 q}\right|}{q\lfloor n / 2\rfloor^{2 q}} .
$$

Consequently, for $n \geq 2$,

$$
(-1)^{q+1}\left(r_{n}^{*}(q)-r_{\lfloor n / 2\rfloor}^{*}(q)\right)>\frac{\left|B_{2 q}\right|}{2 q}\left(\frac{1}{(\lfloor n / 2\rfloor+1)^{2 q}}-\left(1-4^{-q}\right) \cdot \frac{2}{n^{2 q}}\right)
$$

and

$$
(-1)^{q-1}\left(r_{n}^{*}(q)-r_{\lfloor n / 2\rfloor}^{*}(q)\right)<\frac{\left|B_{2 q}\right|}{2 q}\left(\left(1-4^{-q}\right) \frac{2}{\lfloor n / 2\rfloor^{2 q}}-\frac{1}{(n+1)^{2 q}}\right) .
$$

Comparing (13) and (15), we conclude that

$$
r_{q}(n):=R_{q}\left(\left\lfloor\frac{n}{2}\right\rfloor, n\right)=r_{n}^{*}(q)-r_{\lfloor n / 2\rfloor}^{*}(q) \quad(n \geq 2) .
$$

This way we obtained several expressions for $r_{q}(n)$ in Theorem 1 below. Simple calculation shows that the estimate (14) is more accurate for $r_{q}(n)$ than (17), which will not be considered in the sequel. Additionally, (16) tell us that $(-1)^{q+1} r_{q}(n)>0$, for all $q \geq 1$ and $n \geq 3$. Indeed, we consider $\delta_{q}(n):=\frac{1}{(\lfloor n / 2\rfloor+1)^{2 q}}-\left(1-4^{-q}\right) \cdot \frac{2}{n^{2 q}}$, figuring in (16). Obviously, $\delta_{q}(2)<0$, for $q \geq 1$. But, for any $q \geq 1$ and $n=3$ we have

$$
\delta_{q}(3)>0 \Leftrightarrow \frac{1}{4^{q}}>\frac{\left(1-4^{-q}\right) \cdot 2}{9^{q}} \Leftrightarrow\left(\frac{9}{4}\right)^{q}>\left(1-4^{-q}\right) \cdot 2,
$$


where $\left(\frac{9}{4}\right)^{q}=\left(1+\frac{5}{4}\right)^{q}>1+q \cdot \frac{5}{4}>2$, for $q \geq 1$. Similarly we confirm also the estimate $\delta_{q}(4)>0$, true for $q \geq 1$.

In case $n \geq 5$ and $q \geq 1$ we obviously have

$$
\delta_{q}(n) \geq \frac{1}{(n / 2+1)^{2 q}}-\left(1-4^{-q}\right) \cdot \frac{2}{n^{2 q}}=: \delta_{q}^{*}(n),
$$

where

$$
\delta_{q}^{*}(n)>0 \Leftrightarrow\left[2\left(1-\frac{2}{n+2}\right)\right]^{2 q}>\left(1-4^{-q}\right) \cdot 2,
$$

with

$$
\left[2\left(1-\frac{2}{n+2}\right)\right]^{2 q} \geq\left[2\left(1-\frac{2}{7}\right)\right]^{2}=\frac{100}{49}>2, \quad \text { for } n \geq 5 .
$$

Considering (13), (15), (18), (14) and (17), we confirm the following result.

Theorem 1. For every integer $n \geq 2$ and any integer $q \geq 1$ we have

$$
H_{n}^{*}=\ln 2+\frac{(-1)^{n+1}}{4\left\lfloor\frac{n+1}{2}\right\rfloor}+\sum_{i=1}^{q-1} \frac{\left(4^{i}-1\right) B_{2 i}}{(2 i)\left(2\left\lfloor\frac{n+1}{2}\right\rfloor\right)^{2 i}}+r_{q}(n),
$$

where

$$
0 \leq(-1)^{q+1} r_{q}(n)<\frac{\left|B_{2 q}\right|}{2 q \cdot\left\lfloor\frac{n+1}{2}\right\rfloor^{2 q}}<2 \frac{\exp \left(\frac{1}{24 q}\right)}{1-2 \cdot 4^{-q}} \sqrt{\frac{\pi}{q}}\left(\frac{q}{e \pi\left\lfloor\frac{n+1}{2}\right\rfloor}\right)^{2 q} .
$$

For $n \geq 3$ and $q \geq 1$ there holds the following improved estimate:

$$
(-1)^{q+1} r_{q}(n)>\frac{\left|B_{2 q}\right|}{2 q}\left(\frac{1}{\left(\left\lfloor\frac{n+1}{2}\right\rfloor+1\right)^{2 q}}-\left(1-4^{-q}\right) \cdot \frac{2}{\left(2\left\lfloor\frac{n+1}{2}\right\rfloor\right)^{2 q}}\right)>0 .
$$

Setting $q=1$ and considering the equality $B_{2}=\frac{1}{6}$, from Theorem 1 we obtain the first simple formula given in the next corollary.

Corollary 1. For any integer $n \geq 2$,

$$
\begin{aligned}
\ln 2+ & \frac{(-1)^{n+1}}{4\left\lfloor\frac{n+1}{2}\right\rfloor}+\frac{1}{12}\left(\frac{1}{\left(\left\lfloor\frac{n+1}{2}\right\rfloor+1\right)^{2}}-\frac{3}{8\left\lfloor\frac{n+1}{2}\right\rfloor^{2}}\right) \\
& <H_{n}^{*}<\ln 2+\frac{(-1)^{n+1}}{4\left\lfloor\frac{n+1}{2}\right\rfloor}+\frac{1}{12\left\lfloor\frac{n+1}{2}\right\rfloor^{2}} .
\end{aligned}
$$

Corollary 2. For an integer $n \geq 15$,

$$
\ln 2+\frac{(-1)^{n+1}}{4\left\lfloor\frac{n+1}{2}\right\rfloor}+\frac{1}{24\left\lfloor\frac{n+1}{2}\right\rfloor^{2}}<H_{n}^{*}<\ln 2+\frac{(-1)^{n+1}}{4\left\lfloor\frac{n+1}{2}\right\rfloor}+\frac{1}{12\left\lfloor\frac{n+1}{2}\right\rfloor^{2}} .
$$

Inspecting the inequalities directly for $n \leq 15$ and using Corollary 2 we confirm the next estimates.

๑For $q=1$ we can prove it directly and for $q \geq 2$ using the Bernoulli inequality. 
Corollary 3. For any integer $n \geq 1$ we have

$$
\begin{aligned}
\frac{3}{5} & <\ln 2+\frac{1}{2(n+1)}+\frac{1}{6(n+1)^{2}} \\
& <H_{n}^{*}<\ln 2+\frac{1}{2(n+1)}+\frac{1}{3(n+1)^{2}}<\frac{6}{5}, \quad \text { for odd } n \\
\frac{2}{5} & <\ln 2-\frac{1}{2 n}+\frac{1}{6 n^{2}}<H_{n}^{*}<\ln 2-\frac{1}{2 n}+\frac{1}{3 n^{2}}<\frac{4}{5}, \quad \text { for even } n
\end{aligned}
$$

Putting $q=2$ in Theorem 1 and considering the equalities $B_{2}=\frac{1}{6}$ and $B_{4}=$ $-\frac{1}{30}$, and examining the estimates for $n=1$ directly, we get the following result.

Corollary 4. For every integer $n \geq 1$,

$$
\ln 2+\frac{(-1)^{n+1}}{4\left\lfloor\frac{n+1}{2}\right\rfloor}+\frac{1}{16\left\lfloor\frac{n+1}{2}\right\rfloor^{2}}-\frac{1}{120\left\lfloor\frac{n+1}{2}\right\rfloor^{4}}<H_{n}^{*}<\ln 2+\frac{(-1)^{n+1}}{4\left\lfloor\frac{n+1}{2}\right\rfloor}+\frac{1}{16\left\lfloor\frac{n+1}{2}\right\rfloor^{2}} .
$$

Using $q=6$ in Theorem 1 and considering the equalities $B_{2}=\frac{1}{6}, B_{4}=B_{8}=$ $-\frac{1}{30}, B_{6}=\frac{1}{42}, B_{10}=\frac{5}{66}$ and $B_{12}=-\frac{691}{2730}$, and inspecting the inequalities for $n=1$ directly, we obtain the next result.

Corollary 5. For any integer $n \geq 1$,

$$
\begin{aligned}
H_{n}^{*}= & \ln 2+\frac{(-1)^{n+1}}{4\left\lfloor\frac{n+1}{2}\right\rfloor}+\frac{1}{16\left\lfloor\frac{n+1}{2}\right\rfloor^{2}}-\frac{1}{128\left\lfloor\frac{n+1}{2}\right\rfloor^{4}} \\
& +\frac{1}{256\left\lfloor\frac{n+1}{2}\right\rfloor^{6}}-\frac{17}{4096\left\lfloor\frac{n+1}{2}\right\rfloor^{8}}+\frac{31}{4096\left\lfloor\frac{n+1}{2}\right\rfloor^{10}}+r_{6}(n),
\end{aligned}
$$

where

$$
-\frac{691}{32760\left\lfloor\frac{n+1}{2}\right\rfloor^{12}}<r_{6}(n)<0 \text {. }
$$

Directly from Theorem 1 we get the following consequence.

Corollary 6 (Estimating the tail of the Mercator series for $\ln 2$ ). For integers $n \geq 2$ and $q \geq 1$,

$$
\sum_{k=n+1}^{\infty} \frac{(-1)^{k-1}}{k}=\frac{(-1)^{n}}{4\left\lfloor\frac{n+1}{2}\right\rfloor}-\sum_{i=1}^{q-1} \frac{\left(4^{i}-1\right) B_{2 i}}{(2 i)\left(2\left\lfloor\frac{n+1}{2}\right\rfloor\right)^{2 i}}+\varepsilon_{q}(n)
$$

where

$$
0<(-1)^{q} \varepsilon_{q}(n)<2 \frac{\exp \left(\frac{1}{24 q}\right)}{1-2 \cdot 4^{-q}} \sqrt{\frac{\pi}{q}}\left(\frac{q}{e \pi\left\lfloor\frac{n+1}{2}\right\rfloor}\right)^{2 q} .
$$

Referring to Corollary 3 we have $H_{n}^{*}>\frac{2}{5}$ for $n \geq 1$. Consequently, using (19) and (20) in Theorem 1 we obtain the next corollary. 
Corollary 7. For integers $n \geq 2$ and $q \geq 1$, the approximation

$$
H_{n}^{*} \approx H_{q}^{*}(n):=\ln 2+\frac{(-1)^{n+1}}{4\left\lfloor\frac{n+1}{2}\right\rfloor}+\sum_{i=1}^{q-1} \frac{\left(4^{i}-1\right) B_{2 i}}{(2 i)\left(2\left\lfloor\frac{n+1}{2}\right\rfloor\right)^{2 i}}
$$

has the relative error

$$
\rho_{q}(n):=\frac{H_{n}^{*}-H_{q}^{*}(n)}{H_{n}^{*}}
$$

estimated as

$$
0<(-1)^{q+1} \rho_{q}(n)<5 \frac{\exp \left(\frac{1}{24 q}\right)}{1-2 \cdot 4^{-q}} \sqrt{\frac{\pi}{q}}\left(\frac{q}{e \pi\left\lfloor\frac{n+1}{2}\right\rfloor}\right)^{2 q} .
$$

\section{References}

[1] V. Lampret, A double inequality for a generalized-Euler-constant function, J. Math. Anal. Appl. 381(1) (2011) 155-165.

[2] V. Lampret, Accurate double inequalities for generalized harmonic numbers, Appl. Math. Comput. 265 (2015) 557-567.

[3] A. Sîntămărian, A new proof of estimating the remainder of the alternating harmonic series, Creat. Math. Inform. 21(2) (2012) 221-225.

[4] A. Sîntămărian, Sharp estimates regarding the remainder of the alternating harmonic series, Math. Inequal. Appl. 18(1) (2015) 347-352.

[5] L. Tóth and L. Bukor, On the alternating series $1-\frac{1}{2}+\frac{1}{3}-\frac{1}{4}+\cdots$, J. Math. Anal. Appl. 282 (2003) 21-25. 